\title{
The organization of the pig T-cell receptor gamma (TRG) locus provides insights into the evolutionary patterns of the TRG genes across Cetartiodactyla
}

\author{
Giovanna Linguiti ${ }^{1}$, Francesco Giannico ${ }^{2}$, Pietro D'Addabbo ${ }^{1}$, Angela Pala ${ }^{1}$, Anna Caputi Jambrenghi ${ }^{3}$, Salvatrice \\ Ciccarese $^{1}$, Serafina Massari ${ }^{4}$ and Rachele Antonacci ${ }^{1, *}$ \\ 1 Department of Biology, University of Bari “Aldo Moro”, Bari, Italy; giovanna.linguiti@uniba.it; \\ pietro.daddabbo@uniba.it; angela.pala@uniba.it; salvatricemaria.ciccarese@uniba.it; \\ rachele.antonacci@uniba.it \\ 2 Department of Veterinary Medicine, University of Bari “Aldo Moro”, Bari, Italy; francesco.giannico@uniba.it \\ 3 Department of Agricultural and Environmental Science, University of Bari "Aldo Moro", Bari, Italy; an- \\ na.caputijambrenghi@uniba.it \\ 4 Department of Biological and Environmental Science and Technologies, University of Salento, Lecce, Italy; \\ sara.massari@unisalento.it \\ * Correspondence: rachele.antonacci@uniba.it;
}

\begin{abstract}
The domestic pig (Sus scrofa) is a species representative of the Suina, one of the four suborders within Cetartiodactyla. In this paper, we reported our analysis of the pig TRG locus in comparison with the loci of species representative of the Ruminantia, Tylopoda and Cetacea suborders. The pig TRG genomic structure reiterates the peculiarity of the organization of Cetartiodactyla loci in TRGC "cassettes", each containing the basic V-J-J-C unit. Eighteen genes arranged in four TRGC cassettes, form the pig TRG locus.

All the functional TRG genes were expressed, and the TRGV genes preferentially rearrange with the TRGJ genes within their own cassette, which correlates the diversity of the gamma-chain repertoire with the number of cassettes.

Among them, the TRGC5, located at the $5^{\prime}$ end of the locus, is the only cassette that retains a marked homology with the corresponding TRGC cassettes of all the analyzed species. The preservation of the TRGC5 cassette for such a long evolutionary time presumes a highly specialized function of its genes, which could be essential for the survival of species. Therefore, the maintenance of this cassette in pigs confirms that it is the most evolutionarily ancient within Cetartiodactyla, and it has undergone a process of duplication to give rise to the other TRGC cassettes in the different artiodactyl species in a lineage-specific manner.
\end{abstract}

Keywords: pig genome; gamma/delta T-cell; TRG locus; TRG genes; gamma/delta high species; Cetartiodactyla; Immunogenomics; evolution

\section{Introduction}

Artiodactyla represents the largest order of terrestrial mammals. In the past, Artiodactyla was considered monophyletic and was traditionally divided into three major lineages: Suina, Tylopoda, and Ruminantia [1]. The modern phylogeny, based on molecular and paleontological data [2-4], recognized that Cetacea, a highly specialized mammalian order that includes whales, dolphins and porpoises, is a nested member of Artiodactyla. Therefore, Cetartiodactyla has become the generally accepted name for the superorder containing both orders, and it currently includes over 330 species grouped into 23 families and 131 genera [5].

However, the phylogeny of this superorder is controversial regarding the relationships among the major clades of cetartiodactyls. Different studies indicate a certain instability at the root of the phylogeny, varying between Suina or Tylopoda in stating the 
first lineage to diverge [4,6-11], or, alternatively, postulating a monophyletic clade containing Tylopoda and Suina as the sister group to Cetacea and Ruminantia [6,12].

Comparative phylogenomic studies, coupled with the development of genomic assemblies for the species belonging to this superorder, can help to resolve the controversies.

Among genomic regions, the complex loci encoding for the T-cell receptor (TR) chains provide valuable resources for the analysis of specific traits and comparative studies in the various species of Cetartiodactyla.

Two separate lineages, characterized as either $\alpha \beta$ or $\gamma \delta$ T lymphocytes according to the expression of TR on their membranes, comprise the total T-cell pool in mammals. In contrast to $\alpha \beta \mathrm{TR}$, which only recognize antigens as peptide fragments bound to major histocompatibility complex (MHC) molecules, a feature known as MHC restriction, $\gamma \delta \mathrm{T}$ cells recognize antigens in their native forms and are not MHC-restricted. The $\gamma \delta$ T cells were initially identified in humans and mice, and no obvious conservation of $\gamma \delta$ T-cell subsets, based on TR repertoire and function, between the two species was found, leading to the notion that human and mouse $\gamma \delta$ T cells are highly different [13]. $\gamma \delta$ T cells have also been studied in animals other than humans and mice.

In artiodactyl species, $\gamma \delta \mathrm{T}$ cells represent a set of the $\mathrm{T}$ population whose characteristics are diverse, and, as in humans and mice, their functions in these species have not yet been well defined [13]. For example, they comprise up to $60 \%$ of the circulating lymphocytes in young cattle, sheep and swine, and, although the percentage decrease with age, they still represent up to $30 \%$ in adults. $\gamma \delta$ T cells also account for up to $35 \%$ of the blood lymphocytes in new-born and young camels, and although the proportion is lower than that in other artiodactyl species, they are still a major population in the blood [14]. Therefore, ruminants and pigs, as well as camels, are $\gamma \delta$ T-cell "high-species" with respect to humans and mice, which are defined as $\gamma \delta \mathrm{T}$ "low species" because of the low percentage (approximately $5 \%$ ) of $\gamma \delta$ T cells in the peripheral blood [13].

Moreover, the potential diversity of the $\gamma$ and $\delta$ chain repertoires is wider and more diverse in the artiodactyl species compared to the restricted and limited repertoire in humans and mice. In turn, the $\gamma$ and $\delta$ chain repertoires depend on the gene organization, which interestingly shows high variability among different species [13, 15-18].

The $\gamma$ and $\delta$ chains are encoded by separate multigene families. Each chain is generated by somatic rearrangements of non-contiguous germline genes belonging to the variable $(V)$, diversity ( $D$; only for the $\delta$ chain) and joining $(J)$ gene types organized in complex TR genomic loci that lie, for each chain, in specific chromosomal regions. During recombination, one of the multiple $V, D$ and $J$ genes is selected and they are joined together to form the variable portion of the chain. After transcription, the $\mathrm{V}-(\mathrm{D})-\mathrm{J}$ sequence is spliced to the constant $(C)$ gene encoding for the constant portion of the chain. The random insertion and deletion of nucleotides at the rearrangement positions create junctional diversity in the highly variable complementarity determining region 3 (CDR3), which is primarily responsible for the antigen recognition. Two other, more variable regions (CDR1 and CDR2) are encoded by the germline $\mathrm{V}$ genes.

The genomic organization of the locus encoding for the TR $\gamma$ chain (TRG) is the most considerably different across vertebrates and seems to be related to the evolution of species $[13,17]$.

In cetartiodactyl species (sheep, goats, cattle, dromedaries and dolphins), the genomic structure of the TRG locus is characterized by the presence of a basic structural scheme, consisting of a V-J-J-C unit or "cassette" [13]. The basic unit also includes, at the $5^{\prime}$ end of the first J gene, a promoter for the germline transcription, and at the $3^{\prime}$ end of the $C$ gene, an enhancer-like element, which controls the local recombinational accessibility.

The number of cassettes varies in the different species as a consequence of reiterated duplications, with a single cassette in dolphins, three in dromedaries and six (sheep) or seven (goats and cattle) in ruminants, arranged in the last in two distinct TRG loci that map separately on the same chromosome [13]. 
In this study, we investigated on the genomic organization of the TRG locus in Sus scrofa, a species that we considered representative of the Suina suborder, to understand the origin and evolutionary dynamics of the TRG loci in the cetartiodactyl group. Our comparative data provide new insights into the evolutionary patterns of the TRG genes within Cetartiodactyla, but also implications for clarifying the phylogenetic relationships among the major clades of this important group of mammals.

Moreover, an expression assay on spleen RNA sample revealed that all the annotated functional TRG genes contributed to the pig $\gamma$ chain repertoire. Each TRGV gene preferentially rearranges with the TRGJ genes of its own cassette, and the V-J region is spliced to the relevant TRGC in the mature transcripts. Therefore, the number of cassettes seem to be relevant for the level of diversity of the $\gamma$ chain repertoire.

\section{Materials and Methods}

\subsection{Pig genome analysis}

To determine the pig TRG locus location, the Sscrofa11.1 genome sequence (GenBank accession: GCA_000003025.6), released by the International Swine Genome Sequencing Consortium, was searched using the BLAST algorithm. A sequence of 317,500 bp was retrieved directly from the reference sequence NC_010451.4 (Sus scrofa chromosome 9 genomic sequence) available at NCBI from 108,654,001 to 108,971,498 (complement) positions. Particularly, the analysed region extended from the AMPH to the STARD3NL genes, found flanking the TRG locus of most mammalian species (https://www.imgt.org/IMGTrepertoire/LocusGenes/\#h1 6).

All TRG genes within the genome sequence were identified and annotated using the available pig TRG cDNA collection (Acc. numbers: AB185441-AB185447) and other artiodactyl TRG genomic sequences as a reference. The beginning and end of each coding exon were identified with accuracy by the presence of splice sites or flanking recombination signal (RS) sequences of the $\mathrm{V}$ and J genes.

The locations of the TRG genes are provided in Supplementary Table S1 together with the position of AMPH and STARD3NL genes.

Moreover, computational analysis of the pig TRG locus was conducted using the RepeatMasker for the identification of genome-wide repeats and low complexity regions (Smit, A.F.A., Hubley, R., Green, P. RepeatMasker open-4.0. at http://www.repeatmasker.org; accessed on 27 May 2021) and Pipmaker [19] for the alignment of the determined pig sequence with itself. The inspection of the obtained dot-plot matrix allowed us to identify portions of the sequence that align with more regions within the sequence itself.

For the identification of the regulatory regions in the pig TRG locus, the nucleotide sequences of $598 \mathrm{bp}$ and $771 \mathrm{bp}$, corresponding respectively to the human $5^{\prime} \mathrm{J} \gamma$ promoter (PJ) and 3' enhancer element (En), were retrieved from the human TRG genomic sequence (Accession no. NC_01045: pos. $38276916-38276319$ for PJ and pos. 38233337-38232567 for En) and compared with the pig corresponding sequences by Clustal Omega [20] at www.ebi.ac.uk.

For the genomic analysis of the Cetoartiodactyla TRGC cassettes, each sequence, which comprises the first TRGV gene till the enhancer-like element, was retrieved from each TRG locus of the different species. For simplicity, we considered the cassette sequences derived from a single representative species for each suborder of Cetartiodactyla, i.e., sheep for Ruminantia, pig for Suina, dromedary for Tylopoda and dolphin for Cetacea. The position of the TRG genes was annotated in Genbank format by SnapGene Viewer utility (SnapGene Viewer 5.3.2, available at www.snapgene.com). The annotated sequences were compared each other by Mauve, a genome multiple alignment software (Mauve 2.4.0, available at darlinglab.org/mauve/mauve.html). 
The functionality of the $\mathrm{V}, \mathrm{J}$ and $\mathrm{C}$ genes was predicted through the manual alignment of sequences adopting the following parameters: (a) identification of the leader sequence at the $5^{\prime}$ of the $\mathrm{V}$ genes; (b) determination of proper RS sequences located at $3^{\prime}$ of the V (V-RS) and 5' of the J (J-RS), respectively; (c) determination of conserved acceptor and donor splicing sites; (d) estimation of the expected length of the coding regions; (e) absence of frameshifts and stop codons in the coding regions of the genes. Conversely, a germline gene is qualified as ORF (open reading frame) if the coding region has an open reading frame, but alterations have described in the splicing sites and/or RS sequences, and/or in changes of conserved amino acids. Finally, a germline gene is qualified as pseudogene (P) if its coding region has stop codon(s) and/or frameshift mutation(s). According to the germline sequences, four TRGV genes are pseudogenes: TRGV4, TRGV6, TRGV11 and TRGV12-2. However, we have considered the TRGV6 (with frameshift in L-PART1 and no-canonical donor splice site) and TRGV12-2 (with frameshift in V-exon) functional genes and modified their nucleotide sequence based on the sequence of productive cDNA clones (GenBank Accession N ${ }^{\circ}$ AB185445 and AB185447) that retain the two genes (Supplementary Table S1).

The TRGV genes were grouped in different subgroups based on the percentage of nucleotide identity by using the Clustal Omega alignment tool, which is available at the EMBL-EBI website (http://www.ebi.ac.uk/ (accessed on 6 May 2021), adopting the criterion that sequences with a nucleotide identity of more than $75 \%$ in the coding region of a TR V gene (i.e., L-PART1+V-EXON) belong to the same subgroup [21].

The TRGJ genes were named by a number in accordance with the name of the belonging TRGC cassette, followed by a hyphen and a number corresponding to their position within the cassette.

The TRGC genes, classified on the basis of the homology with the ruminant corresponding genes, define the name to the cassettes. All pig TRGC genes were predicted to be functional, even the TRGC4 gene, whose EX1 is out of frame in the assembly probably due to a sequence error (Supplementary Table S1). In fact, the sequence of the first exon of the pig TRGC4 gene lacks a " $\mathrm{c}$ " nucleotide in position 114, which is present in the same gene sequence detected in a previous genomic assembly (Sscrofa10.2) and in a cDNA clone (GenBank accession no. L21161).

\subsection{Phylogenetic analysis}

The human, bovine, sheep, dolphin TRGV and TRGC gene sequences used for the phylogenetic analysis, as annotated, were retrieved from the IMGT ${ }^{\circledR}$ database (IMGT Repertoire, http://www.imgt.org, IMGT/GENE-DB, [22]. The goat, dromedary and pig gene sequences were retrieved from GenBank database with the following accession numbers: NC_030811.1 (goat TRG locus contig as characterized by Giannico et al. [17]); GCA_000803125.1, JN165102 and JN172913 (dromedary TRG locus as characterized by Antonacci et al. [23]); and NC_010451.4 (pig TRG locus as characterized in this work). We combined the nucleotide sequences of the V-REGION of the pig TRGV genes with the corresponding gene sequences of sheep, goats, cattle, dromedaries, dolphins and humans. Similarly, the pig TRGC gene nucleotide sequences as well as their 3'UTR nucleotide sequences (from the stop codon to the poly-A site) were aligned with the corresponding ruminant, dromedary and dolphin gene sequences.

Multiple alignments of the gene sequences under analysis were carried out with the MUSCLE program [24]. The evolutionary analyses were conducted in MEGA X [25,26]. We used the neighbour-joining (NJ) method to reconstruct the phylogenetic tree [27]. The evolutionary distances were computed using the p-distance method [28] and are in the units of the number of base differences per site.

Based on the TRGC phylogenetic tree, the RelTime method [29,30] was employed to compute the mean substitution rates along each branch and estimate the species divergence time. The chicken TRGC gene sequence was specified as outgroup. The TimeTree was computed using one constraint to estimate absolute times. We choose a time range of 66 and $68 \mathrm{Ma}$, which represent the occurrence of divergence between dromedary (Ty- 
lopoda) and pig (Suina), as calibration point. The time range derived from previous estimation based on fossil records and molecular data [11].

\subsection{5' rapid amplification of $c D N A$ ends ( $R A C E) P C R$}

Total RNA was extracted from spleen of an adult animal using the Trizol method according to the manufacturer's protocol (ThermoFisher Scientific). Approximately 5 ug of RNA was reverse transcribed with Superscript II (ThermoFisher Scientific) by using an oligo-dT adapter primer.

After linking a poly-C tail at the $5^{\prime}$ end of the ss cDNA, two different amplification experiments were set up to perform the ds cDNA with Platinum Taq polymerase (ThermoFisher Scientific). In one PCR reaction, GL1L (5' TCCAGAAGACAAAGGTATGTTCCA 3') was used as the lower primer, which was designed on a conserved nucleotide sequence of the first exon shared by the pig TRGC3, TRGC4 and TRGC6 genes. The second PCR experiment was performed using as the lower primer GL1Lbis (5' TCAAGAAGACAAAGATGTGTCCCA $3^{\prime}$ ), designed on the sequence of the first exon of the pig TRBC5 gene. In both PCR reactions an anchor oligonucleotide was used as the upper primer (AAP) provided by the supplier (ThermoFisher Scientific). The PCR conditions were as follows: $30 \mathrm{~s}$ at $94^{\circ} \mathrm{C}, 45 \mathrm{~s}$ at $55^{\circ} \mathrm{C}$, and $1 \mathrm{~min}$ at $72^{\circ} \mathrm{C}$ for 35 cycles. The products were then both amplified in a subsequent nested PCR experiment by using GL2L /AUAP primer pair. GL2L (5' TATYTCAGCAATYGAAGGAAG 3', where Y is A or $G)$ is designed on a sequence upstream of GL1L as well as GL1Lbis; while, the AUAP oligonucleotide was provided by the supplier (Invitrogen). The PCR conditions were as follows: $15 \mathrm{~s}$ at $94^{\circ} \mathrm{C}, 15 \mathrm{~s}$ at $60^{\circ} \mathrm{C}, 15 \mathrm{~s}$ at $72^{\circ} \mathrm{C}$ for 30 cycles. The final cycle was extension for $30 \mathrm{~min}$ at $72^{\circ} \mathrm{C}$.

The RACE products were then gel-purified and cloned using the StrataClone PCR Cloning Kit (Stratagene). Random selected positive clones for each cloning were sequenced by a commercial service. cDNA sequence data were processed and analysed using the Blast program (http://www.blast.ncbi.nlm.nih.gov/Blast.cgi), the Clustal Omega alignment tool (http://www.ebi.ac.uk/) and IMGT tools [IMGT/V-QUEST [31, 32] with integrated IMGT/JunctionAnalysis tools [33,34] and the IMGT unique numbering for the $\mathrm{V}$ domain [35] (http://www.imgt.org/).

All cDNA clones were registered in EMBL database with the Accession numbers from OL906430 to OL906434.

\section{Results}

\subsection{Genomic structure of the TRG locus in Sus scrofa}

The public genomic assembly Sscrofa11.1 was analysed in order to isolate the TRG locus in pigs, a species that was considered as the reference for the Suina suborder of Artiodactyla in our subsequent comparative analyses. To this end, the pig TRG locus was characterised and annotated according to the rules of the international IMGT database (IMGT®, http://www.imgt.org).

A sequence of approximately $317 \mathrm{~kb}$ was recovered. The TRG genes occupy about $113 \mathrm{~kb}$ and are flanked by the AMPH and STARD3NL genes at the $5^{\prime}$ and $3^{\prime}$ ends, respectively (Figure 1). 


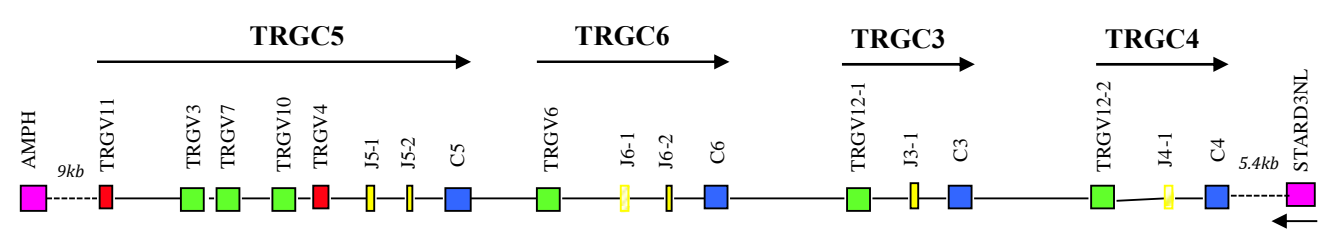

$\underline{4 \mathrm{~kb}}$

\begin{tabular}{|c|c|}
\hline \multicolumn{2}{|l}{ Legend: } \\
$\square$ V-GENE Functional \\
$\square$ & V-GENE Pseudogene \\
$\square$ & J-GENE Functional \\
J-GENE ORF \\
$\square$ C-GENE Functional \\
$\square$ GENE not related \\
\hline
\end{tabular}

Figure 1. Schematic representation of the genomic organisation of the pig TRG locus deduced from the genome assembly Sscrofa11.1. The name of each TRGC cassette is indicated by arrow. The TRGV6 and TRGV12-2 genes, whose coding sequences are not in frame within the assembly, are indicated in the map as functional since they were found in productive cDNA clones (see text). The diagram shows the position of all related and unrelated TRG genes according to nomenclature. The boxes representing the genes are not to scale. The exons are not shown. The arrow indicates the transcriptional orientation of the STARD3NL gene.

Basically, the deduced genomic structure of the TRG locus in pigs reflects the peculiarity of the organization of this region in the other artiodactyl species, consisting of a set of closely related "cassettes", each containing the V-J-J-C basic unit arranged in the same transcriptional orientation $[13,17]$. Particularly, the pig TRG locus comprises eight TRGV, six TRGJ and four TRGC genes distributed in four V-J-(J)-C cassettes, which is fewer than the number of cassettes characterizing the ruminant TRG locus (seven in cattle and goats, and six in sheep) $[13,17]$, but it is greater than the number present in the dromedary (three cassettes) [23] and dolphin (a single cassette) [36] loci. The total number of TRG genes in pigs is also lower than that of ruminants (36 in goats, 34 in cattle and 32 in sheep), but comparable to that in dromedaries (17 genes) and higher than that in dolphins (six genes). The pig cassettes were classified, proceeding from the $5^{\prime}$ to the $3^{\prime}$ end of the region, as TRGC5, TRGC6, TRGC3 and TRGC4, in accordance with the corresponding ruminants' cassettes (see the paragraph below for details; IMGT®, http://www.imgt.org). Five TRGV genes are in the TRGC5 cassette, and only one is in each of the other three cassettes. Two TRGJ genes compose the TRGC5 and TRGC6 cassettes, while only one TRGJ gene is present in the TRGC3 as well as in the TRGC4 cassette. Finally, each cassette comprises a TRGC gene that is assigned the same name as the cassette.

\subsection{Gene analysis}

The TRGV genes were assigned to seven different subgroups by nucleotide-sequence identity (see Section 2). All the subgroups consist of a single TRGV gene except for the TRGV12 subgroup, which consists of two genes.

Six are predicted to be functional genes as defined by the IMGT rules (see Section 2; IMGT®, http://www.imgt.org), and only two are pseudogenes (Supplementary Table S1). The structure of the pig germline TRGV genes is shown in Supplementary Figure S1A.

The classification of the pig TRGV genes to the subgroups was established by a phylogenetic analysis in the context of the Cetartiodactyla superorder by comparing all the pig gene sequences with the corresponding ruminant (goat, sheep and cattle), dromedary and dolphin gene sequences. The human genes were also included in the analysis. Thus, the V-REGION nucleotide sequences of all the selected TRGV genes were combined in the same alignment, and an unrooted phylogenetic tree was constructed using the NJ method [27] (Figure 2). 


\section{$x$}

Figure 2. The neighbour-joining (NJ) tree inferred from the pig, sheep, goat, bovine, dromedary, dolphin and human TRGV gene sequences. The evolutionary analyses were conducted in MEGA X $[25,26]$. The optimal tree with the sum of branch length $=5.56210337$ is shown. The percentage of replicate trees in which the associated taxa clustered together in the bootstrap test (100 replicates) is shown next to the branches [37]. The tree is drawn to scale, with branch lengths in the same units as those of the evolutionary distances used to infer the phylogenetic tree. The evolutionary distances were computed using the p-distance method [28] and are in the units of the number of base differences per site. This analysis involved 45 nucleotide sequences. Codon positions included were $1 s t+2 n d+3 r d+$ Noncoding. All ambiguous positions were removed for each sequence pair (pairwise deletion option). There was a total of 349 positions in the final dataset.

Each pig TRGV gene is marked with a red circle. The branches highlighted by the letters group mammalian genes described in the text. The gene functionality according to IMGT rules (F: functional, ORF: open reading frame, P: pseudogene) is indicated. The IMGT 6-letter for species (Susscr, Bostau, Oviari, Caphir, Camdro, Turtru and Homsap) standardized abbreviation for taxon is used.

The tree shows that each pig TRGV subgroup forms a monophyletic group with corresponding ruminant genes and, when present, with corresponding genes of dromedaries, dolphins and humans, consistent with the birth of each pig subgroup from a mammalian common ancestor prior to the divergence of the different species. Two major groupings of the mammalian genes are clearly distinguishable in the tree. The branch A groups genes exhibiting a conserved nature across species, with a clear orthology. This branch includes all the TRGV genes belonging to the artiodactyl TRGC5 cassette [13]. Therefore, each pig TRGV gene subgroup was named in accordance with each corresponding artiodactyl gene subgroup name, i. e., TRGV3, TRGV4, TRGV7, TRGV10 and TRGV11. The artiodactyl TRGC5 cassette has been shown to be the most evolutionarily ancient [13]. It would have been duplicated to generate a second one that developed the other artiodactyl TRGC cassettes, whose TRGV genes are all included in branch B, where they form three principal groupings, C, D and E (Figure 2). In C, all the ruminant genes 
belonging to distinct subgroups (TRGV2, TRGV8 and TRGV9) and located in the TRGC3 (named TRGC7 in cattle) cassette are clustered. Only one pig TRGV gene and the dromedary TRGV1 gene are closely related to these genes as paraphyletic branches. Hence, in the absence of a perfect (univocal) orthology, we classified the pig gene as belonging to a new subgroup. It was named TRGV12-1 since the pig TRGV12 subgroup consists of two genes. The pig TRGV12-2 gene groups with paralogous genes located in the ruminant TRGC4, TRGC1 and TRGC2 cassettes (D branch). Finally, the E branch groups the TRGV genes belonging to the ruminant TRGV6 subgroup and located in the TRGC6 cassette together with the orthologous pig TRGV6 and the dromedary TRGV2 genes. In accordance with these phylogenetic data, we classified the pig TRGC cassettes, proceeding from the $5^{\prime}$ to the $3^{\prime}$ end of the region, as the ruminant TRGC5, TRGC6, TRGC3 and TRGC4.

Supplementary Figure S1B reports the nucleotide and deduced amino-acid sequences of all the pig TRGJ genes identified in the region. The genes are 49-60 bp long and show conservation of the canonical FGXG(A) amino-acid motif, whose presence defines the functionality of $J$ genes. The only exception is the TRGJ4-1 gene, which has a different amino acid in the second position of the motif and was therefore classified as ORF (Supplementary Table S1). Notably, TRGJ4-1 was found to be rearranged within one cDNA clone (GenBank accession no. AB185447). Each TRGJ gene is flanked by a 12 RS at the $5^{\prime}$ end and by a donor splice site at the $3^{\prime}$ end. The RS are well conserved in the crucial positions with respect to the consensus sequence, except for the first and second nucleotide of the "cac" sequence of the heptamer of the TRGJ6-1 gene that was classified as ORF.

The structure of the pig TRGC genes is similar to that of the homologous mammalian genes (http://www.imgt.org/IMGTrepertoire/Proteins/) (Supplementary Figure S1C). The first exon (EX1) encodes the $C$ domain, while the first part of the connecting region is encoded by one (EX2A) or three (EX2A, EX2B and EX2C) exons. The remaining portion of the connecting region, the transmembrane region and the cytoplasmic region are encoded by EX3. Hence, the connecting region of the pig TRGC genes differs in length and amino-acid sequence depending on the encoding EX2 exons. This heterogeneity is shared with the TRGC genes of other artiodactyl species, where the connecting region can be encoded by three exons, two exons or only one exon [13]. In particular, the pig TRGC5 consists of only one EX2, as observed in ruminants, dromedaries and dolphins. Moreover, in pigs as in camels, the TRGC gene consisting of two EX2, is missing compared to that in ruminants.

\subsection{5'RACE assay}

To evaluate the features of the expressed $\gamma$ chain repertoire with respect to the germline genes identified in the genome assembly, we performed a 5' RACE assay on an RNA sample isolated from the spleen of an adult animal. After the reverse transcription, two different amplification experiments were set up. In the first, we used GL1L as a lower primer designed based on a conserved sequence of the first exon shared by the TRGC3, TRGC4 and TRGC6 genes. In the second, the lower primer GL1Lbis, designed on the first exon of the TRGC5 gene, was used. The two amplification products were separately cloned, and 35 randomly selected positive clones for each cloning were sequenced. Sequence analysis revealed that all the clones consisted of rearranged $\mathrm{V}-\mathrm{J}-\mathrm{C}$ transcripts of different length, but most of them were redundant cDNAs. Five different transcripts were obtained. Four cDNAs (GL2L6, GL2L13, GL2L17 and GL2L36) were isolated from the first amplification experiment, and one clone (GL1L2) from the second one (Supplementary Figure S2).

Each sequence was manually analysed to identify the TRGV, TRGJ and TRGC genes through alignment with the germline pig genes annotated in the assembly. Supplementary Figure S2 shows the protein display of these cDNA sequences together with further cDNA clones from pig spleen retrieved from the IMGT database (accession no. 
AB185441-AB185447). The comparison of the expressed sequences with the germline ones allowed us to ascertain that all the cDNA perfectly matched with the corresponding germline TRGV, TRGJ and TRGC sequences, with the exception of a single mismatch in the TRGV6 gene sequences, which results in an amino-acid change ( $\mathrm{T}$ instead of $\mathrm{N}$ ) in cDNAs (Supplementary Figure S2). In spite of the small collection of cDNA clones, we can say that all the annotated TRGV genes but one (TRGV11 pseudogene) are used in the $\mathrm{V}-\mathrm{J}$ somatic recombination for the $\gamma$ chain. Even the TRGV4 gene, classified as an in-frame pseudogene, is included. Likewise, all the TRGJ genes are used except for the TRGJ6-1 gene, which presents no canonical RS sequence, accounting for the lack of transcription.

Finally, the sequence analysis of the constant portion in each cDNA revealed the presence of the TRGC gene proximal to the TRGJ gene used in the V-J rearrangement.

Therefore, the sequence analysis of the cDNAs confirms, as in other artiodactyl TRG loci, that each TRGV gene preferentially rearranges with the TRGJ genes of its own cassette, and the V-J region is spliced to the relevant TRGC in the mature transcripts $[38,39]$, leading to the conclusion that the cassette number is crucial for the diversity of the $\gamma$ chain repertoire. However, the number of cassettes in pigs seems to limit the potential gene recombinations compared to other artiodactyls. Indeed, in ruminants, the greater number of TRG genes distributed within a greater number of reiterated cassettes increases the potential rearrangements $[13,17]$. The level of diversity of the pig $\gamma$ chain repertoire is lower not only than that of ruminants but also than that of dromedaries. As a matter of a fact, recent studies [23,40-41) have provided evidence that the somatic hypermutation mechanism (SHM) contributes to the expansion of the diversity of the dromedary $\gamma$ TR repertoire, even though only three cassettes lie in the locus.

However, a recent deep expression study [39] performed in different pig lymphoid tissues and T-cell populations confirms that about $75 \%$ of the rearrangements were within a cassette, but there was also trans-cassette V-J recombination and VJ-C trans-cassette splicing. Curiously, the last two mechanisms do not seem to occur for the TRGC5 cassette, where most of the TRGV genes are located.

\subsection{Genomic architecture and identification of the regulatory elements in the pig TRG cassettes}

The genomic architecture of the pig TRG locus was further investigated, aligning the masked corresponding sequence (from the TRGV11 to TRGC4 gene) against itself with the Pipmaker program (Figure 3). The dot-plot matrix detects the occurrence of internal homology units along the entire pig TRG region, as indicated by dots and lines. Parallel lines clearly show the mode of evolution of the locus by tandem duplications of $\mathrm{V}-\mathrm{J}-\mathrm{J}-\mathrm{C}$ cassettes. Three lines parallel to the perfect main diagonal line, which indicates the match of each base of the sequence with itself, highlight the high level of nucleotide identity between the four pig TRGC cassettes. Each parallel line is interrupted due to the lack of nucleotide identity between the TRGV-gene-containing regions of the different cassettes. Therefore, units of internal homology are detected just between the J-C gene blocks (the blue squares in Figure 3). The only exception is the clear homology between the TRGV12 subgroup genes, which results in the longest homology unit consisting of a TRGJ6-1/TRGC6/TRGV12-1/TRGJ3-1/TRGC3/TRGV12-2 gene block (blue dashed square in Figure 3). The high homology between the TRGC3 and TRGC4 gene cassettes, even within their TRGV region, supports the idea that they may arise from a recent duplication event. 


\section{Sus scrofa}

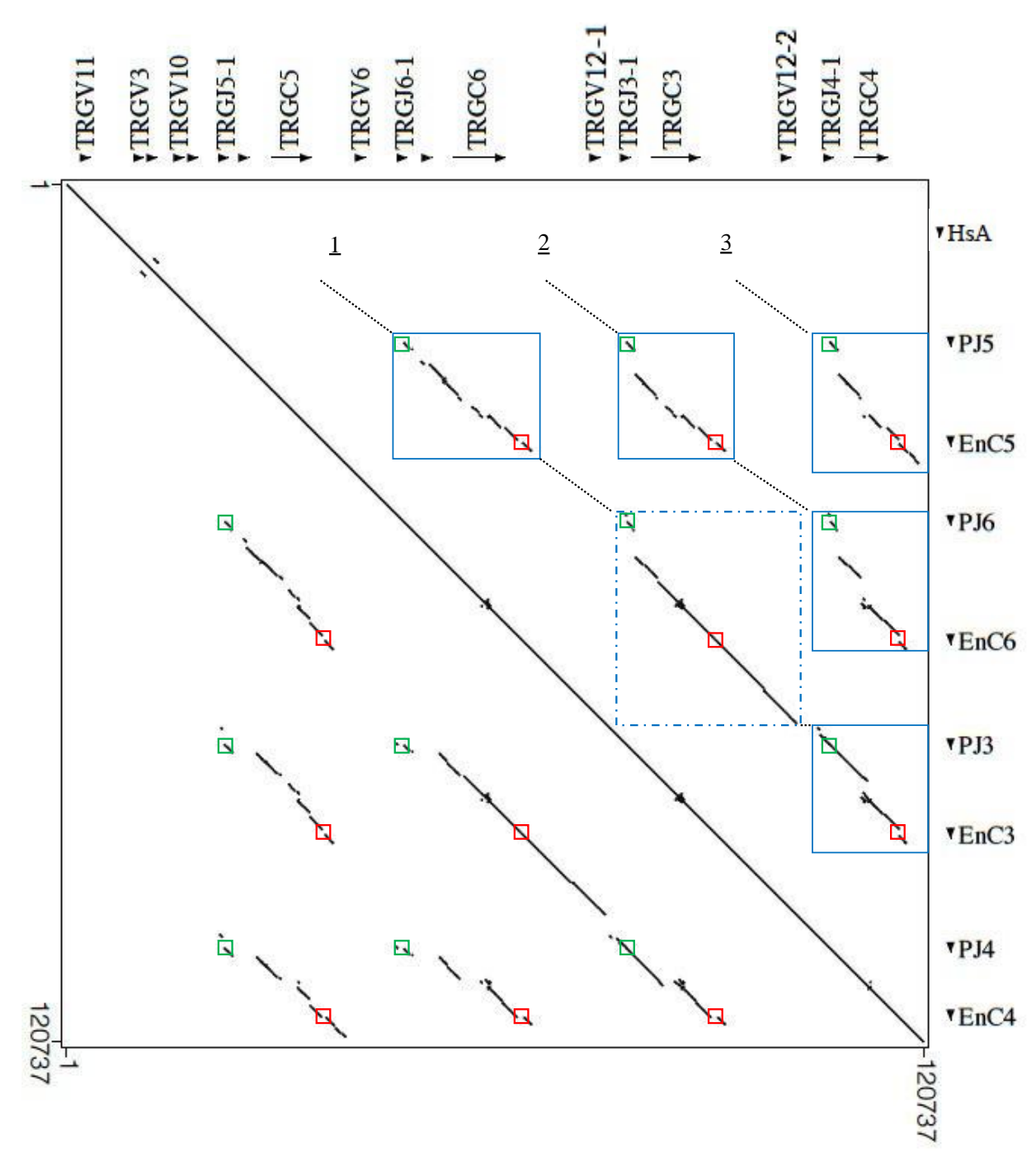

Figure 3. Dot-plot of the pig TRG locus sequence against itself. The transcription orientation of each sequence is indicated by arrows and arrowheads. Three parallel lines (1-3) to the perfect main diagonal line indicate the internal homology units between the TRGC cassettes. The interruptions of the parallel lines are indicated by dotted lines. The blue boxes show the internal homology of the J-C gene blocks. The blue dashed square indicates the longest duplicated region containing the TRGJ6-1/TRGC6/TRGV12-1/TRGJ3-1/TRGC3/TRGV12-2 gene block.

It should be noted that the lines of similarity corresponding to each $\mathrm{J}-\mathrm{C}$ gene block extend beyond the TRGJ and TRGC coding regions, comprising the germline transcription promoters (PJ) located at the $5^{\prime}$ end of each TRGJ gene distal to the relevant TRGC gene (green boxes in Figure 3), and the enhancer-like elements (En) positioned about $2 \mathrm{~kb}$ from the 3' ends of all the TRGC genes (red box in Figure 3), respectively. These regions have been experimentally determined in humans $[42,43]$ as the cis-control elements that cooperate to regulate the accessibility of the TRG locus to the site-specific recombination machinery through their possible interaction with STAT5 proteins. A comparison between the human cis-control elements and the corresponding pig sequences revealed high conservation in the pig sequences of $400 \mathrm{bp}$ in the PJ (Supplementary Figure S3A) and $153 \mathrm{bp}$ in the En (Supplementary Figure S3B) regions, where STAT consensus motifs (TTCNNNGAA) are located. Therefore, the functional value of the PJ and En regions is 
underlined by their conservation, which led to the need for them to duplicate themselves together with the rest of the cassettes.

\subsection{Phylogenetic relationships of the Cetoartiodactyla TRGC genes}

The availability of the pig germline sequences allowed us to estimate the timing and mode of TRGC gene duplications along the evolutionary history of cetartiodactyl species. We constructed a timetree by aligning the coding nucleotide sequences of the pig TRGC genes with the corresponding ruminant (goat, sheep and cattle), dromedary and dolphin ones. The chicken TRGC gene sequence was used as the outgroup. For the calibration of the phylogenetic tree (evolutionary time), we used the time of divergence between dromedaries and pigs. The tree highlights that the evolutionary relationship of the TRGC genes in the different species is mostly consistent with the current phylogeny. As a matter of a fact, all the sequences, except for the TRGC5 pig-related genes, make lineage-specific clusters, which show consistency between the estimated divergence time and the accepted phylogenetic divergence time of each species.

In detail, the origin of the TRGC genes within the Cetartiodactyla superorder seems to have started from a common ancestral gene, which would have duplicated approximately 70 Mya ago, as indicated by node 1, which resolves the cetartiodactyl TRGC genes in two principal paraphyletic groups (A and B in Figure 4).

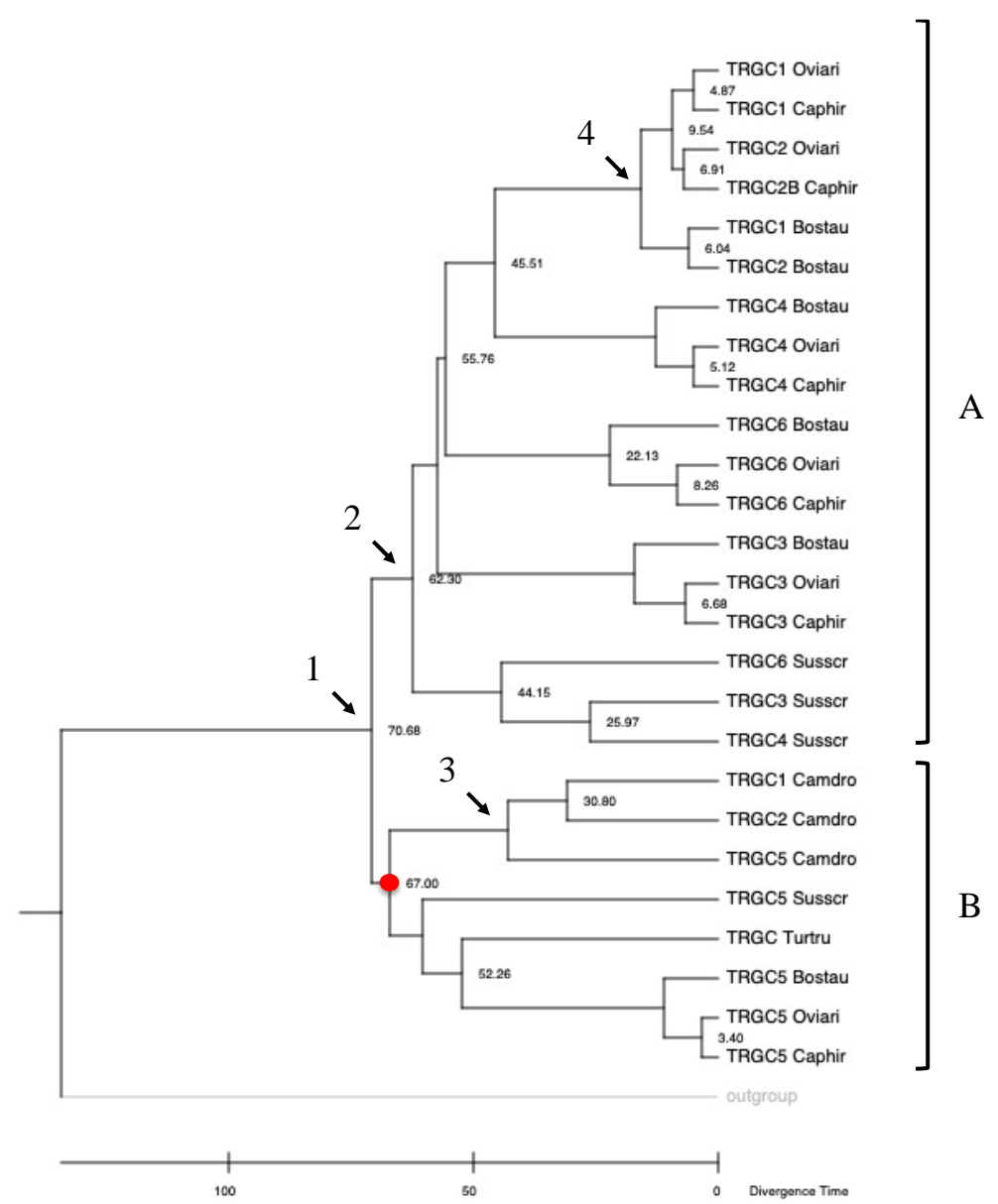

Figure 4. Time-calibrated phylogenetic tree of TRGC genes in Cetartiodactyla derived from MEGAX [25] applying RelTime method. This analysis involved 27 nucleotide sequences. Codon positions included were 1st+2nd+3rd+Noncoding. All ambiguous positions were removed for each sequence pair (pairwise deletion option). There were a total of 818 positions in the final dataset. The calibration point (red circle) is arranged on node to indicate the occurrence of divergence between Tylopoda (Camdro) and Suina (Susscr) estimated on fossil records and molecular data [11]. The chicken TRGC sequence was used as outgroup. A (ruminant and pig TRGC genes) and B 
(dromedary and dolphin TRGC genes) represent the two major paraphyletic groups. Nodes 1-4 are described in the text.

The IMGT 6-letter for species (Susscr, Bostau, Oviari, Caphir, Camdro, Turtru and Homsap) standardized abbreviation for taxon is used.

In A, only ruminant and pig TRGC genes are present. Therefore, the first TRGC duplicative event would have occurred only in Suina and Ruminantia lineages and, consequently, after Tylopoda and Cetacea divergence. Differently, the most evolutionarily ancient TRGC gene (the TRGC5 pig-related gene) is still maintained across all the species, including dromedaries and dolphins, as indicated by the monophyletic branch B. In fact, it groups the pig, ruminant and camel TRGC5 and the dolphin TRGC genes. It is evident that strong selective pressure has maintained this ancient gene in the different cetartiodactyl genomes for a long period of evolutionary time. The high homology of this gene in the different species, which conserves a clear orthology, is certainly related to a functional constraint of its protein product.

Furthermore, differently from the dolphin TRGC, which remained unique during evolution, the dromedary TRGC5 gene gave rise, approximately $50 \mathrm{Mya}$, to the birth of the TRGC1 and TRGC2 genes as indicated by the species-specific clustering (node 3 in Figure 4). The evolution of the dromedary TRGC genes, as a group apart from the other Cetartiodactyla suborders, would support phylogenomic research about an early divergence of Tylopoda $[8,44]$.

Likewise, in branch A, the ruminant and pig TRGC genes form distinct clades. They originated from the duplicated ancestral gene (the TRGC6 pig-related gene) that would have duplicated itself approximately $63 \mathrm{Mya}$, after Suina and Ruminantia speciation (node 2 in Figure 4). Therefore, subsequent duplication events gave rise to the TRGC3 and TRGC4 genes within pig lineage distinctly from the TRGC1, TRGC2, TRGC3, TRGC4 and TRGC6 genes generated in the ruminant lineage (Figure 4). All the ruminant TRGC genes appear to have evolved from a common ancestor, as they maintain a clear orthology between sheep, goats and cattle, except for the TRGC1 and TRGC2 genes, for which the evolutionary duplications seem to have occurred independently within Bovinae and Caprinae subfamilies (node 4 in Figure 4).

We also investigated the evolutionary behaviour of the flanking region of the TRGC genes with respect to their coding regions. The 3'UTR nucleotide sequences of the TRGC genes were aligned and a phylogenetic tree was constructed using the NJ method (Figure 5). The results are consistent with the previous ones. The tree perfectly recapitulates the phylogeny of the Cetartiodactyla species, grouping the sequences in lineage-specific monophyletic branches. In contrast to the coding sequences whose homology between species is preserved, the evolutionary history of their flanking regions shows a divergent pattern that groups them within lineage-specific clusters. Similarly, the flanking regions of the sheep as well as goat TRGC1 and TRGC2 genes seem to have evolved in a species-specific manner (Figure 5) with respect to their coding regions that still maintain a clear homology between species (Figure 4). 


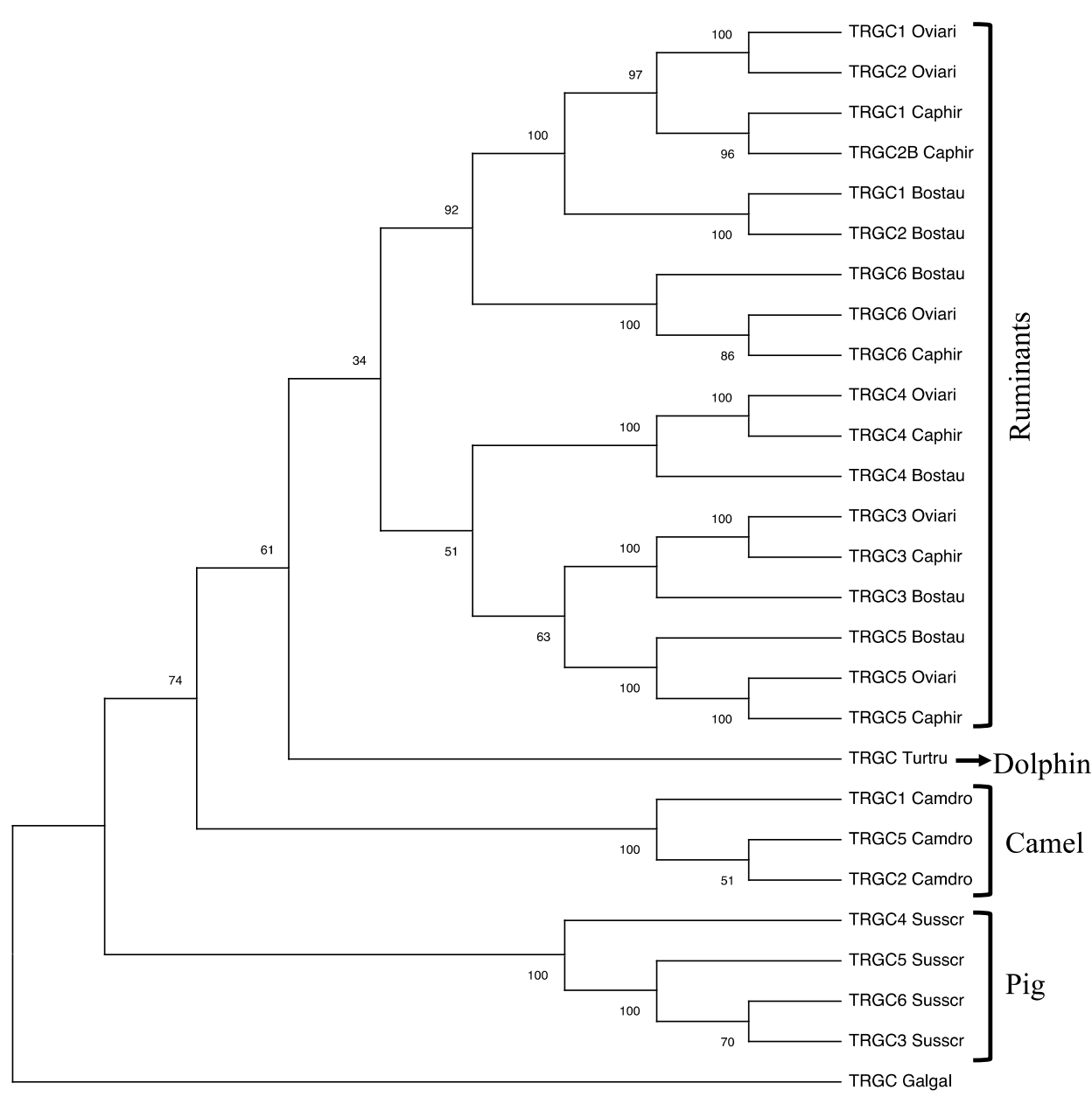

Figure 5. The NJ tree inferred from the pig, sheep, goat, bovine, dromedary and dolphin 3'UTR nucleotide sequences of the TRGC genes. The evolutionary analyses were conducted in MEGA X $[25,26]$. The optimal tree with the sum of branch length $=1.76269382$ is shown. The percentage of replicate trees in which the associated taxa clustered together in the bootstrap test (100 replicates) are shown next to the branches [37]. The tree is drawn to scale, with branch lengths in the same units as those of the evolutionary distances used to infer the phylogenetic tree. The evolutionary distances were computed using the p-distance method [28] and are in the units of the number of base differences per site. This analysis involved 27 nucleotide sequences. Codon positions included were $1 \mathrm{st}+2 \mathrm{nd}+3 \mathrm{rd}+$ Noncoding. All ambiguous positions were removed for each sequence pair (pairwise deletion option). There were a total of 727 positions in the final dataset.

The brackets highlight lineage-specific monophyletic branches. The IMGT 6-letter for species (Susscr, Bostau, Oviari, Caphir, Camdro, Turtru and Homsap) standardized abbreviation for taxon is used.

\subsection{Phylogenetic relationships of the Cetoartiodactyla TRGC cassettes}

We completed our genomic analysis by comparing the entire sequences of all the TRGC cassettes retrieved within each TRG locus from different cetartiodactyl species. Each sequence starts from the ATG codon of the first TRGV gene located at the $5^{\prime}$ end of the cassette and ends with the enhancer-like element at the $3^{\prime}$ end. Only one representative species per Cetartiodactyla suborders was selected. The cassettes' nucleotide sequences for the selected pig, sheep, dromedary and dolphin TRG loci were first combined in the same alignment, and an unrooted phylogenetic tree was constructed using the NJ method [27] (left part of Figure 6). 

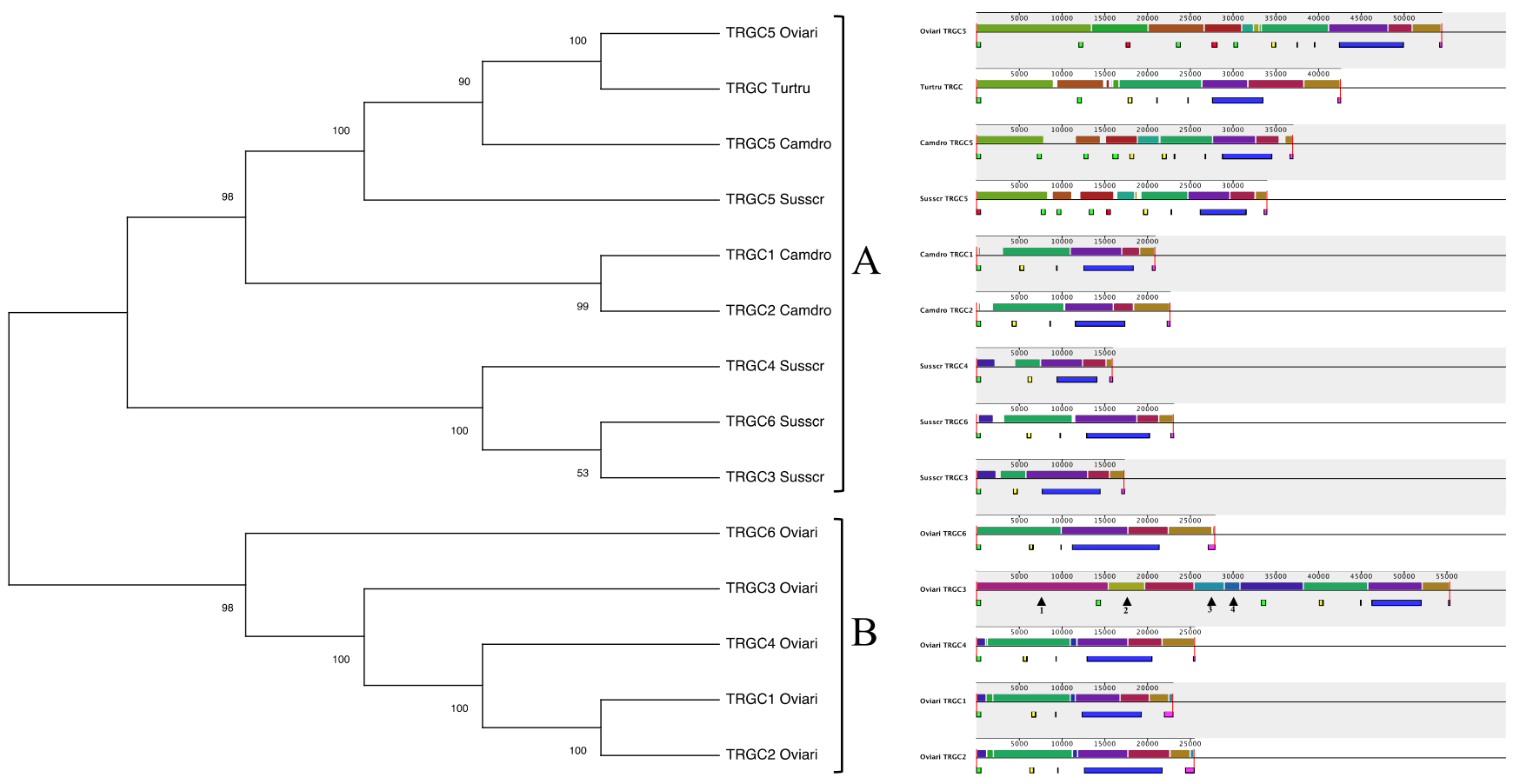

Figure 6. Comparison of Cetartiodactyla TRGC cassettes. Left, the NJ tree inferred from the TRGC cassette sequences retrieved from pig, sheep, dromedary and dolphin TRG loci. The evolutionary analyses were conducted in MEGA X [25,26]. The optimal tree with the sum of branch length = 1.91796264 is shown. The percentage of replicate trees in which the associated taxa clustered together in the bootstrap test (100 replicates) are shown next to the branches [37]. The evolutionary distances were computed using the p-distance method [28] and are in the units of the number of base differences per site. This analysis involved 15 nucleotide sequences. Codon positions included were $1 \mathrm{st}+2 \mathrm{nd}+3 \mathrm{rd}+$ Noncoding. All ambiguous positions were removed for each sequence pair (pairwise deletion option). There were a total of 127184 positions in the final dataset. Right, schematic representation of the nucleotide comparison of the same genomic region. Coloured blocks highlight syntenic regions between the different sequences. Blocks below the line indicate the position of TRGV (green, yellow and red for functional, ORF and pseudogenes, respectively), PJ-TRGJ genes (black line), TRGC genes (blue) and En elements (pink) is reported.

The black arrows are described in the text.

The tree resolves the sequences in two main branches (A and B). Generally, all the cassette sequences group in a species-specific manner. The only exceptions are the pig, sheep and dromedary TRGC5 cassettes and the dolphin TRGC single one, which form a monophyletic group within branch A, highlighting how the high level of conservation during the evolution of the different lineages affected not only the TRGC (Figure 4) and the TRGV (Figure 2) genes present in those cassettes but the entire genomic sequences of the same cassettes. This confirms, once again, the ancient origin of these cassettes.

Similarly to the relative TRGC genes (Figure 4), the dromedary TRGC1 and TRGC2 cassettes are closely related to this conserved cassette group. Furthermore, in branch A, the pig cassette sequences form a sister group with Tylopoda and the TRGC5 cassette group, while the relative TRGC genes seem to be more related to Ruminantia genes (Figure 4). Instead, the Ruminantia cassettes sequences group in a separated branch (branch B in Figure 6).

The phylogenetic analysis of the TRGC cassettes is in accordance with an overall visual inspection of their sequences (right part of Figure 6). The TRGC cassette sequences were multi-aligned for an overview, by a computational method, of the homologous regions, which are highlighted by blocks of identical colours.

Blocks with identical colors are more evident between phylogenetically related cassettes, where even the order of the homologous blocks is conserved along the 
sequences. As an example, the pig TRGC cassettes (except for the TRGC5) that form a monophyletic group in the tree (branch A) are depicted by an identical string of blocks, even if with different sizes, i.e., blue, emerald, purple, brown and dark yellow. Similarly, the ancient cassettes (pig, sheep and dromedary TRGC5 and dolphin TRGC) share homology regions, as the green block located at the $5^{\prime}$ end of each sequences. The differences are also easily detectable, as the presence of an additional emerald block within the sheep TRGC5 cassette and/or the diverse size of shared blocks due to the duplication events that occurred during the sequence evolution of each species. In this context, the sheep TRGC3 cassette sequence is depicted by four coloured blocks not shared with any other sequence.

However, conserved blocks of homology are shared across all the cassettes, as the purple block containing the TRGC genes, the emerald block representative of the TRGV genes, the dark yellow block located at the end of each sequence, etc., showing that an evolutionary pressure to preserve the general structure of the cassettes exists.

\section{Discussion}

Among the elements of vertebrate adaptive immunity, $\gamma \delta \mathrm{T}$ cells represent an enigmatic population of immune cells whose role is yet to be fully elucidated. However, their unique features in $\gamma \delta$ "high species" make animals such as pigs valuable models for broadening our understanding of $\gamma \delta$ T-cell biology and for investigating the evolution of immune capacities.

In this context, the availability of a long-read pig genome assembly provided us with the opportunity to analyze, from an evolutionary point of view, the TRG genes within the Cetartiodactyla superorder.

First, using the current reference genome Sscrofa 11.1, we determined the genomic organization and gene content of the pig TRG locus.

During the preparation of this work, a publication became available that includes the annotation of the porcine TRG locus in three different genomic assemblies [39]. One of these is the current Sscrofa11.1 analysed in our work. Nevertheless, we considered it appropriate to include, in this article, our annotation data deduced from Sscrofa11.1 because they were established in accordance with the ruminant gene names and the rules of the IMGT nomenclature (IMGT®, http://www.imgt.org). This allowed us to more easily perform comparative analyses with the other species sequences, mostly retrieved from the international IMGT database.

The pig TRG structure reflects the peculiarity of the corresponding cetartiodactyls' loci, organized in $\mathrm{V}-\mathrm{J}-(\mathrm{J})-\mathrm{C}$ cassettes $[13,17]$. The dolphin locus represents the simplest one, with the TRG genes arranged in a single cassette, while the pig TRG locus, organized in four $\mathrm{V}-\mathrm{J}-(\mathrm{J})-\mathrm{C}$ cassettes, is in between the ruminant (six or seven cassettes) and camelid (three cassettes) loci for the number of cassettes.

The pig cassettes were classified based on the phylogenetic relationships among the TRGV genes and named as the ruminant corresponding cassettes. This is accordance with the phylogenetic behavior of all the cetartiodactyl TRGV genes, which are grouped on the basis of their belonging to a given cassette. This allowed us to attribute the nomenclature to each pig gene and to observe that, among all, the TRGV genes belonging to the TRGC5 cassette conserved a clearer orthology with the corresponding genes of all the species, including dolphins (Figura 2). Likewise, the TRGC5 is the only pig TRGC gene to maintain conservation among the species, as indicated by the monophyletic group of branch B (Figure 4). Indeed, besides the genes, the entire sequence of the pig TRGC5 cassette, located at the 5' end of the TRG locus, preserves extensive blocks of homology and a tight phylogenetic relationship with the corresponding one in all the species (Figure 6). As a result, the TRGC5 is the only cassette that still retains a marked homology 
with the corresponding TRGC cassettes of all the cetartiodactyl species analyzed in this study.

Therefore, our data confirm that the pig TRGC5 cassette resembles the ancestral cassette that arose before Cetartiodactyla divergence. Its preservation for such a long evolutionary time, implies a highly specialized function of its genes, which could be essential for the survival of the species. For instance, it has been shown that the genes within the TRGC5 cassette would preferentially be expressed in specific gd cell populations in pigs as well as in ruminants $[39,45]$.

The functional constraint involving the coding portion of the corresponding TRGC5 genes of the different species does not involve the non-coding regions ( $3^{\prime}$ UTR) of the same genes, which instead show intraspecies conservation.

During the artiodactyls' evolution, the ancient TRGC5 cassette underwent the first duplicative event that gave rise to the birth of the TRG loci in the different species. Each locus, consisting of reiterated cassettes, was then generated in a lineage-specific mode, as indicated by the three phylogenetic trees.

According to the time tree, the duplication involving the ancestral gene TRGC5 would have been shared by Suina and Ruminantia, supporting Tylopoda as the first lineage to diverge $[7,8]$. However, the evolutionary relationships of the 3 ' UTRs of the TRGC genes show an early divergence of Suina, while Tylopoda precedes the appearance of Cetacea + Ruminantia (Cetruminantia), which is consistent with mtDNA data [11]. This information is also consistent with the phylogenetic relationships of the sequences relative to the entire TRGC5 corresponding cassettes, which form a monophyletic group within branch A (Figure 6). Nevertheless, if one observes the other TRGC cassettes, this latter tree shows a closer relationship between Suina and Tylopoda than with Ruminantia.

Overall, these data seem to confirm a certain instability at the root of the phylogeny of Cetartiodactyla, in line with previously reported results. Certainly, the different results could be related to differences in phylogenetic inference methods, molecular markers, taxon sampling and outgroup choice. However, given the role of the immune-receptor genes linked to the habits of life of each species, our molecular markers might not be so appropriate for completely solving controversial relative to the phylogeny of the Cetartiodactyla superorder.

In conclusion, our study, with the analysis of the pig TRG genes, contributes to the knowledge of the evolution of the TRG locus within Cetartiodactyla, confirming that this TR locus is the most considerably different across vertebrates and seems to be related to the evolution of species. The great plasticity supports the need to investigate the TRG genomic organization in as many species as possible to provide insight into the unique features of the $\gamma \delta$ T cells.

Supplementary Materials: The following are available online at www.mdpi.com/xxx/s1, Figure S1: A) Structure of the pig TRGV genes based on the IMGT Protein display. The deduced amino acid sequences of the TRGV genes were manually aligned according to IMGT unique numbering for the V-REGION [35] to maximise homology. Only the functional genes and the in-frame pseudogene TRGV4 are shown. All sequences exhibit the typical framework regions (FR) and complementarity determining regions (CDR) and the four amino acids (depicted and indicated in bold): cysteine 23 (1st-CYS) in FR1-IMGT, tryptophan 41 (CONSERVEDTRP) in FR2-IMGT, hydrophobic (here L and F) 89, and cysteine 104 (2nd-CYS) in FR3-IMGT, with the exception of TRGV12-2 gene that lacks the CONSERVED-TRP. Conversely, CDR-IMGT vary in amino acid composition and length. In particular, the CDR1-IMGT was five, eight or nine amino acid lengths (AA) long. The CDR2-IMGT ranged in length from four to eight AA and the germline CDR3-IMGT was four or five AA long. Conversely, FR-IMGT varied above all in amino acid composition. The description of the strands and loops and of the FR-IMGT and CDR-IMGT is according to the IMGT unique numbering for V-REGION [35]. The amino acid length of the CDR-IMGT is also indicated in square brackets. B) Nucleotide and deduced amino acid sequences of the pig TRGJ genes. The consensus sequence of the heptamer and nonamer is provided at the top of the figure and is underlined. The numbering 
adopted for the gene classification is reported on the left of each gene. The donor splice site for each TRGJ is shown. The canonical FGXG amino acid motifs are underlined. No functional TRGJ genes are indicated in italics. C) IMGT Protein display of the pig TRGC genes. The descriptions of the strands and loops were collected according to the IMGT unique numbering for the C-DOMAIN [46]. The extracellular region (C domain) is shown with black letters, the connecting region is in orange, the trans-membrane region is in purple, and the cytoplasmic region is in pink. 1st-CYS C23, CONSERVED-TRP W41 and hydrophobic AA L89 and 2nd-CYS C104 are coloured (IMGT colour menu) and in bold, Figure S2: Protein display of cDNA clones derived from 5' RACE assay (clones series GL) and from IMGT database (clones series AB) in comparison with the corresponding germline TRGV genes. The TRGV, TRGJ and TRGC gene names are listed, respectively, at the left and right of the figure. Leader region (L-REGION), CDR-IMGT and FR-IMGT are indicated according to the IMGT unique numbering for V-DOMAIN. The four amino acids, Cysteine 23 (1st-CYS) in FR1-IMGT, tryptophan 41 (CONSERVED-TRP) in FR2-IMGT, hydrophobic (here L and F) 89, and cysteine 104 (2nd-CYS) in FR3-IMGT, with the exception of TRGV12-2 gene that lacks the CONSERVED-TRP, are highlighted. The name of the clones is also reported. The amino acid change is boxed, Figure S3: Comparison of cis-control elements in the human and pig TRG loci. The nucleotide sequences of the human and pig PJ $\gamma$ germline promoters (A), and enhancers (B) were aligned. In A), the alignment of conserved $400 \mathrm{bp}$ between human and pig PJ $\gamma$ germline promoters was shown. Two conserved motifs for STAT5 proteins, one typical (TTCNNNGAA) and a second atypical (TTCNNNGTA) at $5 \mathrm{bp}$ downstream of the first motif, are shown by red boxes. The J-RS sequences are also boxed. In B), the alignment of conserved $153 \mathrm{bp}$ between human and pig enhancers was shown. The three (NF $2-N F \gamma 4$ ) nuclear protein binding sites protected from DNase I digestion [47], were underlined. NF $\gamma 2-N F \gamma 4$ constitute the minimal enhancer fragment. NF 2 conserves the STAT5 motif; while in NF 33 , the most extensively protected site from DNase I digestion, the conserved PEBP2 transcription factor site was found. The position of the promotor and enhancer regions in the pig TRG locus are reported in Supplementary Table S1, Table S1: Description of the related and no related TRG genes in the Sus scrofa chromosome 9 genome assembly (NCBI Reference Sequence NC_010451.4). The position of all genes and their classification and functionality are reported. The position of the J promoters (PJ) and enhancer elements (EnC) are also inserted.

Author Contributions: Conceptualization, G.L., S.M., and R.A.; methodology, G.L., P.D., A.C.J. and A.P.; investigation, G.L., F.G. and P.D.; writing-original draft preparation, S.M. and R.A.; writing-review and editing, S.M. and R.A.; supervision, S.C.; funding acquisition, R.A. All authors have read and agreed to the published version of the manuscript.

Funding: The financial support of the University of Bari and of University of Salento is gratefully acknowledged. This research was funded by "Contributo di Ateneo per progetti competitivi residuo fondo anno 2015 - (University of Bari).

Institutional Review Board Statement: In this study, the animals were cared for and handled in compliance with the EU legislation on animal welfare regulations (directive 2010/63/EU, which updates and replaces the 1986 directive 86/609/EEC on the protection of animals used for scientific purposes) and following the University's research policies.

Informed Consent Statement: Not applicable.

Data Availability Statement: The data presented in this study are available within the article or supplementary material.

Acknowledgments: The authors would like to acknowledge Mr. Massimo Lacitignola and Dr. Nicolò De Vito for their technical support.

Conflicts of Interest: The authors declare no conflict of interest.

\section{References}

1. Simpson, G.G. The principles of classification and a classification of mammals. Bull. Am. Mus. Natl. Hist. 1945, 85, 1-350. 
2. Graur, D.; Higgins, D.G. Molecular evidence for the inclusion of cetaceans within the order Artiodactyla. Mol. Biol. Evol. 1994, 11,357-364.

3. Montgelard, C.; Catzeflis, F.M.; Douzery, E. Phylogenetic relationships of artiodactyls and cetaceans as deduced from the comparison of cytochrome b and 12S rRNA mitochondrial sequences. Mol. Biol. Evol. 1997,14, 550-559.

4. Gatesy, J.; Milinkovitch, M.; Waddell, V.; Stanhope, M. Stability of cladistic relationships between Cetacea and higher-level Artiodactyl Taxa. Syst. Biol . 1999,48, 6-20.

5. IUCN, 2018. The IUCN Red List of Threatened Species. Version 2018-2. http://www. iucnredlist.org.

6. Matthee, C.A.; Burzlaff, J.D.; Taylor, J.F.; Davis, S.K. Mining the mammalian genome for artiodactyl systematics. Syst. Biol. 2001, 50, 367-390.

7. Zhou, X.M.; Xu, S.X.; Zhang, P.; Yang, G. Developing a series of conservative anchor markers and their application in phylogenomics of Laurasiatherian mammals. Mol. Ecol. Res. 2011, 11, 134-140.

8. Meredith, R.W.; Janecka, J.E.; Gatesy, J.; Ryder, O.A.; Fisher, C.A.; Teeling, E.C.; Goodbla, A.; Eizirik, E.; Simão, T.L.L.; Stadler, T.; Rabosky, D.L.; Honeycutt, R.L., Flynn, J.J.; Ingram, C.M.; Steiner, C.; Williams, T.L.; Robinson, T.J.; Burk-Herrick, A.; Westerman, M.; Ayoub, N.A.; Springer, M.S.; Murphy, W.J. Impacts of the cretaceous terrestrial revolution and KPg extinction on mammal diversification. Science 2011, 334, 521-524.

9. Hassanin, A.; Delsuc, F.; Ropiquet, A.; Hammer, C.; Jansen van Vuuren, B.; Matthee, C.; Ruiz-Garcia, M.; Catzeflis, F.; Areskoug, V.; Nguyen, T.T.; Couloux, A. Pattern and timing of diversification of Cetartiodactyla (Mammalia, Laurasiatheria), as revealed by a comprehensive analysis of mitochondrial genomes. C. R. Biol. 2012, 335, 32-50.

10. Vislobokova, I.A. On the origin of Cetartiodactyla: Comparison of data on evolutionary morphology and molecular biology. Paleontol. J. 2013, 47, 321-334.

11. Zurano, J.P.;Magalhães, F.M.; Asato, A.E.; Silva, G.; Bidau, C.J.; Mesquita, D.O.; Costa, G.C. Cetartiodactyla: Updating a time-calibrated molecular phylogeny. Mol. Phylogenet. Evol. 2019, 133,256-262.

12. Arnason, U.; Gullberg, A.; Gretarsdottir, S.; Ursing, B.; Janke, A. The mitochondrial genome of the sperm whale and a new molecular reference for estimating eutherian divergence dates. J. Mol. Evol. 2000, 50, 569-578.

13. Antonacci, R.; Massari, S.; Linguiti, G.; Jambrenghi, C. A.; Giannico, F.; Lefranc, M.P.; Ciccarese, S. Evolution of the T-Cell Receptor (TR) Loci in the Adaptive Immune Response: The tale of the TRG Locus in Mammals. Genes(Basel) 2020,11, 624.

14. Hussen, J.; Schuberth, H.J. Recent Advances in Camel Immunology. Front. Immunol. 2021, 11, 614150.

15. Antonacci, R.; Lanave, C.; Del Faro, L.; Vaccarelli, G.; Ciccarese, S.; Massari, S. Artiodactyl emergence is accompanied by the birth of an extensive pool of diverse germline TRDV1 genes. Immunogenetics 2005, 57, 254-266.

16. Pégorier, P.; Bertignac, M.; Nguefack Ngoune, V.; Folch, G.; Jabado-Michaloud, J.; Giudicelli, V.; Duroux, P.; Lefranc, M. P.; Kossida, S. IMGT® Biocuration and Comparative Analysis of Bos taurus and Ovis aries TRA/TRD Loci. Genes 2020, 12, 30.

17. Giannico, F.; Massari, S.; Jambrenghi, A.C.; Soriano, A.; Pala, A.; Linguiti, G.; Ciccarese, S.; Antonacci, R. The expansion of the TRB and TRG genes in domestic goats (Capra hircus) is characteristic of the ruminant species. BMC Genomics. 2020, $21,623$.

18. Massari, S.; Linguiti, G.; Giannico F.; D'Addabbo, F; Ciccarese, S.; Antonacci, R. The Genomic Organisation of the TRA/TRD Locus Validates the Peculiar Characteristics of Dromedary $\delta$-Chain Expression. Genes 2021, 12, 544.

19. Schwartz, S.; Zhang, Z.; Frazer, K.A.; Smit, A.; Riemer, C.; Bouck, J.; Gibbs, R.; Hardison, R.; Miller, W. PipMaker-A web server for aligning two genomic DNA sequences. Genome Res. 2000, 10, 577-586.

20. Madeira, F.; Park, Y.M.; Lee, J.; Buso, N.; Gur, T.; Madhusoodanan, N.; Basutkar, P.; Tivey, A.R.N.; Potter, S.C.; Finn, R.D.; Lopez, R. The EMBL-EBI search and sequence analysis tools APIs in 2019. Nucleic Acids Res. 2019, 2, 47(W1):W636-W641.

21. Lefranc, M.-P.; Forster,A.; Baer,R.; Stinson, M.A.; Rabbitts,T.H.Diversity and rearrangement of the human T cell rearranging gamma genes : Nine germ-line variable genes belonging to two subgroups. Cell. 1986, 45, 237-246.

22. Giudicelli, V.; Chaume, D.; Lefranc, M.P. IMGT/GENE-DB: a comprehensive database for human and mouse immunoglobulin and T cell receptor genes. Nucleic Acid Res. 2005, 93, D256-D261.

23. Antonacci, R.; Linguiti, G.; Burger, P.A.; Castelli, V.; Pala, A.; Fitak, R.; Massari, S.; Ciccarese, S. Comprehensive genomic analysis of the dromedary T cell receptor gamma (TRG) locus and identification of a functional TRGC5 cassette. Dev. Comp. Immunol. 2020, 106, 103614.

24. Edgar, R.C. MUSCLE: a multiple sequence alignment with reduced time and space complexity. BMC Bioinformatics $2004,5,113$.

25. Kumar, S.; Stecher, G.; Li, M.; Knynar, C.; Tamura, K. MEGAX: Molecular Evolutionary Genetics Analysis across Computing Platforms. Mol. Biol. Evol. 2018, 35, 1547-1549.

26. Stecher, G.; Tamura, K.; and Kumar S. Molecular Evolutionary Genetics Analysis (MEGA) for macOS. Molecular Biology and Evolution 2020, 37, 1237-1239.

27. Saitou, N.; Nei, M. The neighbor-joining method- a new method for reconstructing phylogenetic trees. Molecular biology and evolution 1987, 4, 406-425.

28. Nei, M.; Kumar, S. Molecular Evolution and Phylogenetics; Oxford University Press: New York,NY, USA, 2000.

29. Tamura, K.; Battistuzzi, F.U.; Billing-Ross, P.; Murillo, O.; Filipski, A.; Kumar, S. Estimating divergence times in large molecular phylogenies. Proc Natl Acad Sci U S A. 2012, 20;109,19333-19338.

30. Tamura, K.; Tao, Q.; Kumar, S. Theoretical Foundation of the RelTime Method for Estimating Divergence Times from Variable Evolutionary Rates. Mol Biol Evol. 2018 1, 35(7), 1770-1782.

31. Brochet, X.; Lefranc, M.P.; Giudicelli, V. IMGT/V-QUEST: the highly customized and integrated system for IG and TR standardized V-J and V-D-J sequence analysis. Nucleic Acids Res. 2008, 36(Web Server issue), W503-508. 
32. Giudicelli, V.; Brochet, X.; Lefranc, M.-P. IMGT/V-QUEST: IMGT standardized analysis of the immunoglobulin (IG) and T celle receptor (TR) nucleotide sequences. Cold Spring Harb. Protoc. 2011, 6, 695-715.

33. Yousfi, M.M.; Giudicelli, V.; Chaume, D.; Lefranc, M.IMGT/JunctionAnalysis: the first tool for the analysis of the immunoglobulin and T cell receptor complex V-J and V-D-J JUNCTIONS. Bioinformatics 2004, 20, i379-i385.

34. Giudicelli, V.; Lefranc, M.-P. IMGT/JunctionAnalysis: IMGT standardized analysis of the V-J and V-D-J junction of the rearranged immunoglobulins (IG) and T cell receptors(TR). Cold Spring Harb. Protoc. 2011, 6, 716-725.

35. Lefranc, M.P.; Pommie, C.; Ruiz, M.; Giudicelli, V.; Foulquier, E.; Truong, L.; Thouvenin-Content, V.; Lefranc, G. IMGT unique numbering for immunoglobulin and $\mathrm{T}$ cell receptor variable domains and Ig superfamily V-like domains. Dev. Comp Immunolog. 2003, 27, 55-57.

36. Linguiti, G.; Antonacci, R.; Tasco, G.; Grande, F.; Casadio, R.; Massari, S.; Castelli, V.; Consiglio, A.; Lefranc, M.P.; Ciccarese, S. Genomic and expression analyses of Tursiops truncatus $\mathrm{T}$ cell receptor gamma(TRG) and alpha/delta (TRA/TRD) loci reveal a similar basic public $\gamma \delta$ repertoire in dolphin and human. BMC Genom. 2016, 17, 634.

37. Felsenstein, J. Confidence limits on phylogenies: An approach using the bootstrap. Evolution 1985, 39, 783-791.

38. Damani-Yokota, P.; Gillespie, A.; Pasman, Y.; Merico, D.; Connelley, T.K.; Kaushik, A.; Baldwin, C.L. Bovine T cell receptors and $\gamma \delta$ WC1 co-receptor transcriptome analysis during the first month of life. Dev. Comp. Immunol. 2018, 88, 190-199.

39. Le Page, L.; Gillespie, A.; Schwartz, J.C.; Prawits, L.M.; Schlerka, A.; Farrell, C.P.; Hammond, J.A.; Baldwin, C.L.; Telfer, J.C.; Hammer, S.E. Subpopulations of swine $\gamma \delta \mathrm{T}$ cells defined by TCR $\gamma$ and WC1 gene expression. Dev. Comp. Immunol. 2021,125,104214.

40. Vaccarelli, G.; Antonacci, R.; Tasco, G.; Yang, F.; El Ashmaoui, H.M.; Hassanane, M.S.; Massari, S.; Casadio, R.; Ciccarese, S. Generation of diversity by somatic mutation in the Camelus dromedarius T-cell receptor gamma (TRG) variable domains. Eur. J. Immunol. 2012, 42, 1-13.

41. Ciccarese, S.; Vaccarelli, G.; Lefranc, M.-P.; Tasco, G.; Consiglio, A.; Casadio, R.; Linguiti, G.; Antonacci, R. Characteristics of the somatic hypermutation in the Camelus dromedarius $\mathrm{T}$ cell receptor gamma (TRG) and delta (TRD) variable domains. Dev. Comp. Immunol. 2014, 46, 300-313.

42. Lee, H.C.; Ye, S.K.; Honjo, T.; Ikuta, K. Induction of germline transcription in the human TCR gamma locus by STAT5. J. Immunol. 2001, 167, 320-326.

43. Wagatsuma, K.; Tani-ichi, S.; Liang, B.; Shitara, S.; Ishihara, K.; Abe, M.; Miyachi, H.; Kitano, S.; Hara, T.; Nanno, M.; Ishikawa, H.; Sakimura, K.; Nakao, M.; Kimura ,H.; Ikuta, K. STAT5 Orchestrates Local Epigenetic Changes for Chromatin Accessibility and Rearrangements by Direct Binding to the TCR $\gamma$ Locus. J. Immunol. 2015,195(4),1804-14.

44. Zhou, X.; Xu, S.; Yang, Y.; Zhou, K.; Yang, G. Phylogenomic analyses and improved resolution of Cetartiodactyla. Mol. Phylogenet. Evol. 2011, 61, 255-264.

45. Gillespie, A.; Yirsaw, A.; Gunasekaran, K.P.; Smith, T.P.; Bickhart, D.M.; Turley, M.; Connelley, T.; Telfer, J.C.; Baldwin, C.L. Characterization of the domestic goat $\gamma \delta \mathrm{T}$ cell receptor gene loci and gene usage. Immunogenetics 2021,73(2),187-201.

46. Lefranc, M.-P.; Pommié, C.; Kaas, Q.; Duprat, E.; Bosc, N.; Guiraudou, D.; Jean, C.; Ruiz, M.; Da Piedade, I.; Rouard, M.; et al. IMGT unique numbering for immunoglobulin and T cell receptor constant domains and Ig superfamily C-like domains. Dev. Comp. Immunol. 2005, 29, 185-203.

47. Spencer, D.M.; Hsiang,Y.H.; Goldman, J.P.; Raulet, D.H. Identification of aT-cell-specific transcriptional enhancer located 3' of C gamma 1 in the murine T-cell receptor gamma locus. Proc. Natl. Acad. Sci. USA 1991, 88, 800-804. 